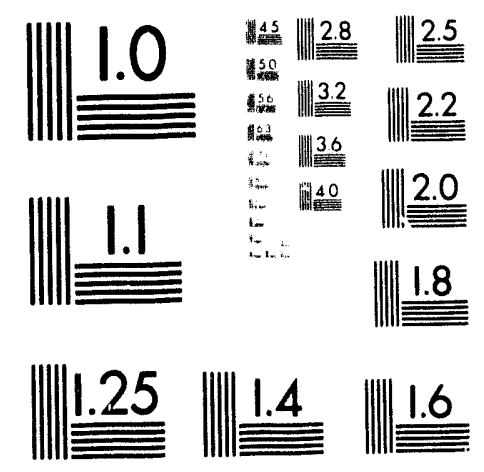



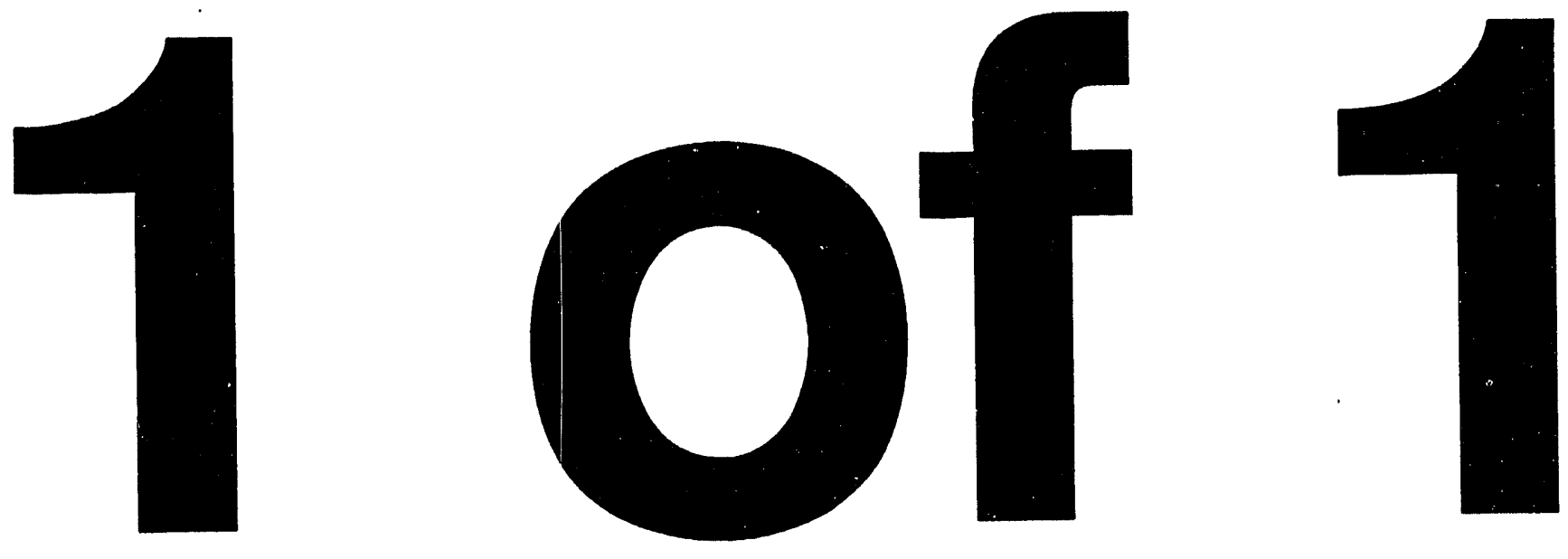


\title{
PERFORMANCE OF \\ THE DIII-D 110 GHz ECH SYSTEM DURING THE FIRST YEAR OF OPERATIONS AND TESTING
}

\author{
by \\ A.L. WRIGHT, J.C. ALLEN, W.P. CARY, \\ and T.E. HARRIS
}

This is a preprint of a paper to be presented at the 15th IEEE/NPSS Symposium on Fusion Engineering, October 11-15, 1993, in Hyannis, Massachusetts, and to be printed in the Proceedings.

Work supported by U.S. Department of Energy Contract DE-AC03-89ER51114

\author{
GENERAL ATOMICS PROJECT 3466 \\ OCTOBER 1993
}

\section{GENERAL

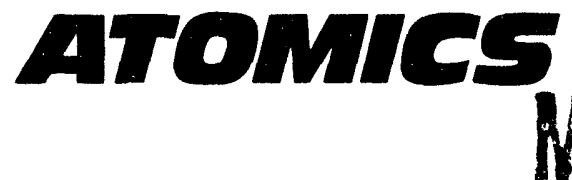




\title{
PERFORMANCE OF THE DIII-D $110 \mathrm{GHZ}$ ECH SYSTEM DURING THE FIRST YEAR OF OPERATIONS AND TESTING*
}

\author{
A.L. Wright, J.C. Allen, W.P. Cary, and T.E. Harris \\ General Atomics \\ P.O. Box 85608, San Diego, California 92186-9784
}

\begin{abstract}
The first of four Varian $500 \mathrm{~kW} 110 \mathrm{GHz}$ gyrotrons (VGT-8011) to be used in the new $2 \mathrm{MW} 110 \mathrm{GHz}$ electron cyclotron heating system being developed for the DIII-D tokamak was put into test at General Atomics within the last year. This gyrotron has been used to demonstrate the overall system efficiency and to validate the design of individual transmission line components. The first plasma heating observe $d$ with a $110 \mathrm{GHz}$ was consistent with the power expected for the greater than $85 \%$ transmission efficiency of $\mathrm{HE}_{1,1}$ power. A comparison of the General Atomics' $\mathrm{TE}_{15,2}$ to $\mathrm{HE}_{1,1}$ mode converter with the Vlasov-type mode converter designed by the University of Wisconsin showed similar conversion efficiencies. The overall ECH system performance during the first year of testing will also be discussed.
\end{abstract}

\section{INTRODUCTION}

The first Varian $110 \mathrm{GHz}$ gyrotron (VGT-8011) was installed on the General Atomics DIII-D tokamak in March 1992. The gyrotron is rated for $500 \mathrm{~kW}$ output power and is the first of four tubes, which when combined will generate $2 \mathrm{MW}$ of $110 \mathrm{GHz}$ electron cyclotron heating for use in physics experiments on the DIII-D tokamak. The operation of the tube has been used to check out the General Atomics transmission line components, two types of mode converters, and the overall system efficiency. The $110 \mathrm{GHz}$ electron cyclotron heating $(\mathrm{ECH})$ system consists of the gyrotron tube. high voltage power supply, superconducting magnets, tank and control electronics, transmission line components, and vacuum system (see Fig. 1 [1]).

\section{SYSTEM OVERVIEW}

The gyrotron is a $110 \mathrm{GHz}, 500 \mathrm{~kW}$ VGT-8011 developed by Varian Microwave Power Tube Division. It is designed to produce high power microwaves in the $\mathrm{TE}_{15,2}$ mode. The $\mathrm{TE}_{15,2}$ mode output travels through a FC-75 cooled sapphire window to a mode converter where the output is converted to an $\mathrm{HE}_{1,1}$ mode for low loss transmission to the tokamak. The gyrotron requires a cathode voltage of $-80 \mathrm{kV}$ dc for operation. This voltage is supplied by a modified neutral beam power supply (MFTF) capable of producing a maximum of $-85 \mathrm{kV} \mathrm{dc}$ and an output current of $80 \mathrm{~A} \mathrm{[2].}$

Superconducting magnets are used to provide the nigh magnetic fields necessary to interact with the electron beam producing an operating cyclotron frequency of $110 \mathrm{GHz}$. The superconducting magnets are encased in a double jacketed $\mathrm{LN}_{2}$ and LHe cryostat. The magnet system consists of eight mag-

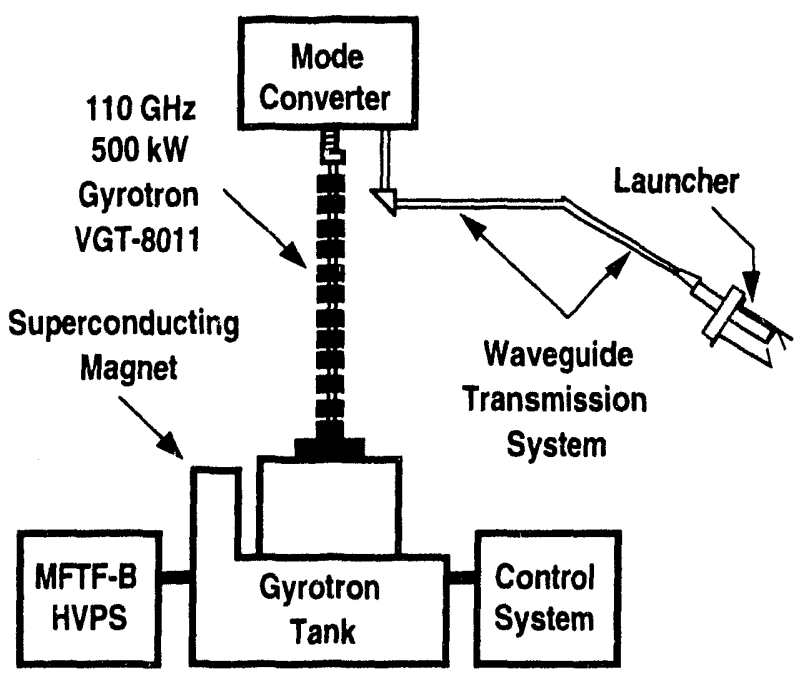

Fig. 1. The $110 \mathrm{GHz}$ ECH System Block Diagram

nets: four co-axial superconducting solenoids for beam shaping and four transverse trim coils used for beam alignment [3].

The gyrotron is supported by a bi-level aluminum tank, filled with transformer oil to reduce high voltage breakdown. The lower high voltage tank was designed to allow for a maximum headroom due to building height restrictions. All electronic monitoring controls and the gun anode voltage regulator are located in the high end of the high voltage tank. The two sealed compartments are interconnected by high voltage feed through bushings. Fiber optic links from the control system in the control room provide control and signal interfaces to the gyrotron tank [3]

The transmission line system consists of the arc detector section, down taper, mode converter, straight sections of waveguide, five miter bends, dc break, and vacuum subsystem (Fig. 2). At the time the transmission line was being designed no viable waveguide window at the $500 \mathrm{~kW}$ level existed; therefore, there is no window at the launcher connected to the tokamak. Because of this, the entire transmission system must be evacuated to the machine of $10^{-8}$ Torr. Since FC-75 is used for window cooling, protection of the tokamak in the event of a window failure requires that the transmission line to have pressure gauges and shutter valves to monitor and protect the machine from contamination by the FC-75.

\section{OPERATIONAL HISTORY}

The $110 \mathrm{GHz}$ ECH system successfully operated into the DIII-D tokamak in March of 1992. Operation into the

*Manuscript received October 11, 1993. This work was supported by the U.S. Department of energy under Contract No. DE-AC03-89ER51114. 


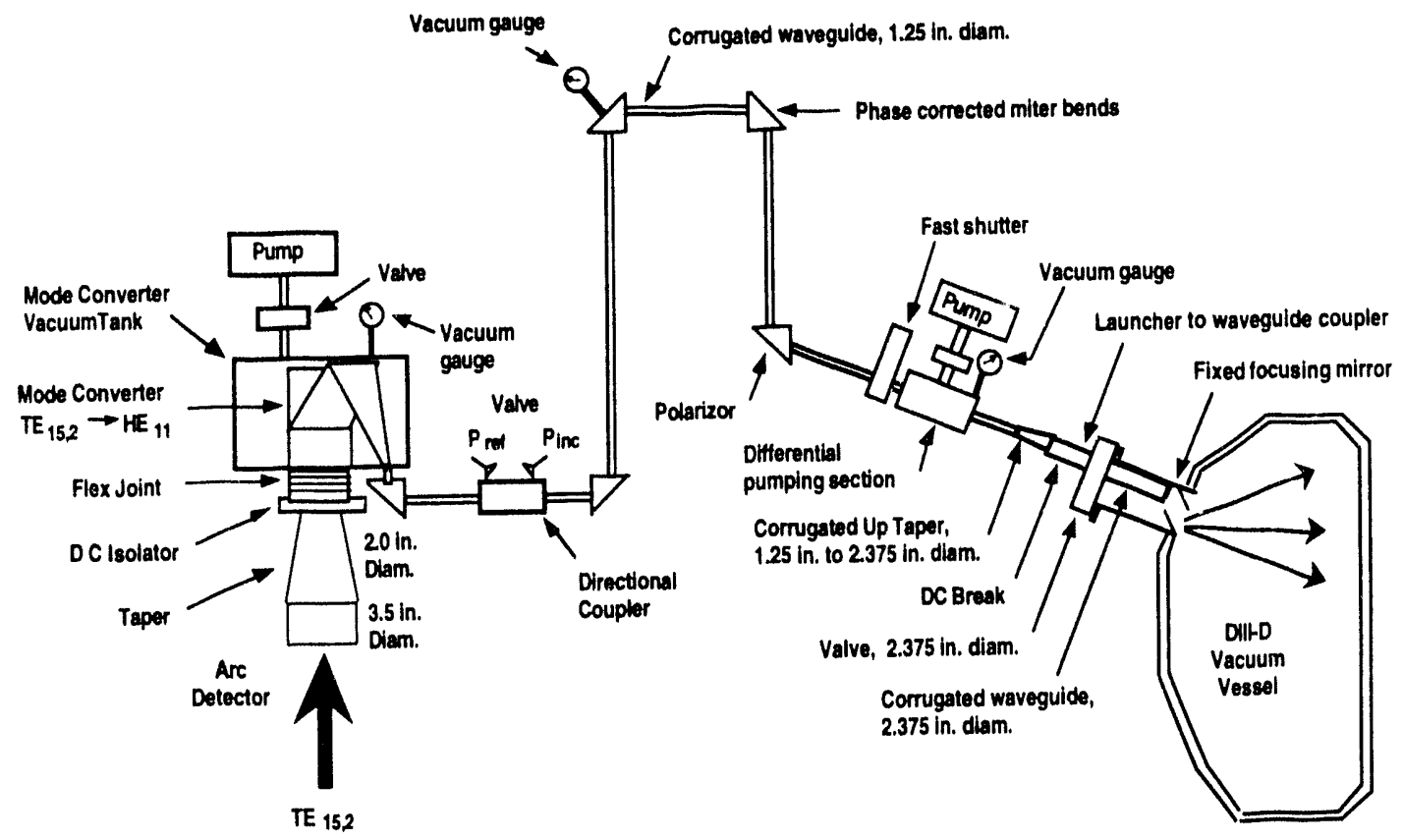

Fig. 2. The transmission line system.

tokamak allowed the entire system to be evaluated. The objective of the transmission line design was to transmit the rf pulse with more than $75 \%$ efficiency. In determining the transmission efficiency one must take into account the ohmic loss of each transmission component as well as any loss due to mode conversion. The total transmission efficiency was calculated by multiplying the individual transmission component efficiencies.

The individual component efficiencies were calculated assuming $100 \% \mathrm{TE}_{15,2}$ mode purity from the gyrotron. However, the effective power that is injected into the tokamak in the $\mathrm{HE}_{1,1}$ mode is dependent upon the mode purity of the microwaves generated by the gyrotron, since power in a mode other than $\mathrm{TE}_{15,2}$ will not be converted into the $\mathrm{HE}_{1,1}$ mode and therefore can not propagate down the transmission line. The percentage of power in the $\mathrm{TE}_{15,2}$ mode multiplied by the transmission efficiency and gyrotron output power will produce the maximum power capable of being injected into the tokamak. To determine the transmission line efficiency laboratory tests (low power) were made on sections of straight waveguide, the mode converter, and miter bends. The efficiency of the mode converter was also confirmed at full operating power of the gyrotron. The results are listed in Table I. The successful injection of power into the tokamak produced a temperature rise in the plasma consistent with the power measured out of the gyrotron and the effective efficiencies of the transmission line components.

The $110 \mathrm{GHz}$ ECH system was operated in a $5 \mathrm{~ms}$ on $10 \mathrm{~ms}$ off multipulse capacity and the temperature rise measured in the plasma was consistent with the power level of approximately $350 \mathrm{~kW}$ measured out of the mode converter into a dummy load.

Because the power level seen in the plasina was less than the expected $500 \mathrm{~kW}$ an effort was made to evaluated why the power level was lower than expected. This led to the exploration of the affect that the taper section has on the gyrotron throughput power. Because of the unexpected result that tapers caused severe degradation in the gyrotron throughput
Table I

Transmission Component Efficiencies

\begin{tabular}{|c|c|c|}
\hline Component & $\begin{array}{l}\text { Measured (m) or } \\
\text { Calculated (c) Loss }\end{array}$ & $\begin{array}{l}\text { Effective } \\
\text { Effective }\end{array}$ \\
\hline Arc detector & $0.2 \% \quad$ (c) & $99.8 \%$ \\
\hline Down taper & $1.5 \% \quad$ (c) & $98.5 \%$ \\
\hline Mode converter (each) & $<100 \%(\mathrm{~m})$ & $>90 \%$ \\
\hline Straight waveguide & $2.0 \% \quad(\mathrm{~m})$ & $98 \%$ \\
\hline Miter bends (5) & $5.5 \% \quad(\mathrm{~m})$ & $94.5 \%$ \\
\hline DC break & $0.5 \% \quad(\mathrm{c})$ & $99.5 \%$ \\
\hline Vacuum pumping tee & $0.05 \% \quad$ (c) & $99.95 \%$ \\
\hline Up taper & $0.15 \% \quad(\mathrm{c})$ & $99.85 \%$ \\
\hline Total transmission & & $81 \%$ \\
\hline
\end{tabular}

efficiency

power, the mode purity of the gyrotron was put into question. A mode separation diagnostic was developed specifically to address the mode purity issue.

\section{GYROTRON SYSTEM PERFORMANCE EVALUATION}

Following the use of the $110 \mathrm{GHz}$ ECH system for initial plasma injection experiments a program was initiated to explore the performance of the mode converter and its possible connection to the low power results. The overall $110 \mathrm{GHz}$ ECH system performance was evaluated through taper testing, mode purity investigation, mode converter efficiency, and collector heat profile testing, details of these tests are described in the following sections.

\section{A. Taper Effects}

The initial objective of the taper testing was to determine the power that was being injected into the mode converter and 
whether or not this had a specific effect on the low throughput power evidenced in earlier tests. The first taper test performed was a double taper configuration (Fig. 3). This test should have quantified the power that was available to the GA mode converter. The result of this taper configuration indicated approximately $450 \mathrm{~kW}$ of throughput power even though the test was performed at gyrotron settings that should have produced a $500 \mathrm{~kW}$ gyrotron output.

In the second taper test, a 1.6-in. diameter taper section was inserted between the double taper configuration of Taper Test \#1 to simulate the worst case constriction inside the GA mode converter. This test resulted in a throughput of approximately $400 \mathrm{~kW}$ with the gyrotron set, as in the first test, to produce a $500 \mathrm{~kW}$ output.

Taper test \#3 was designed to identify whether the low power results in Taper Test \#2 were due to the added constriction of the 1.6-in. taper or to the added length of the taper. A straight 2.0 -in. diameter section the same length as the 1.6-in. taper section in Taper Test \#2 was installed between the double down tapers. The power output was approximately $450 \mathrm{~kW}$. Since the power of the third taper test was approximately the same as the power in the first test, it can be concluded that the power reduction demonstrated in Taper Test \#2 was due to the 1.6-in. taper, however, microwave theory does not predict the observed reduction in power, and there is no consistent theory or model which could explain this power reduction phenomena under the assumption that all the throughput power is in the primary $T E_{15,2}$ mode. These results, therefore, led to questioning the mode purity of the gyrotron.

\section{B. Mode Purity}

A mode separator diagnostic was developed based on GA mode converter technology. The mode separator design eliminated the 1.6-in. taper section of the GA mode converter, thus attempting to minimizing the effect of any additional tapering on mode purity. The mode separator was installed and standard calorimetric methods were used to provide quantitative data on the primary $\mathrm{TE}_{15,2}$ mode power from the gyrotron versus any alternate mode power.

The general trend observed by the mode separator was that as the gyrotron power increased, the percentage of power in

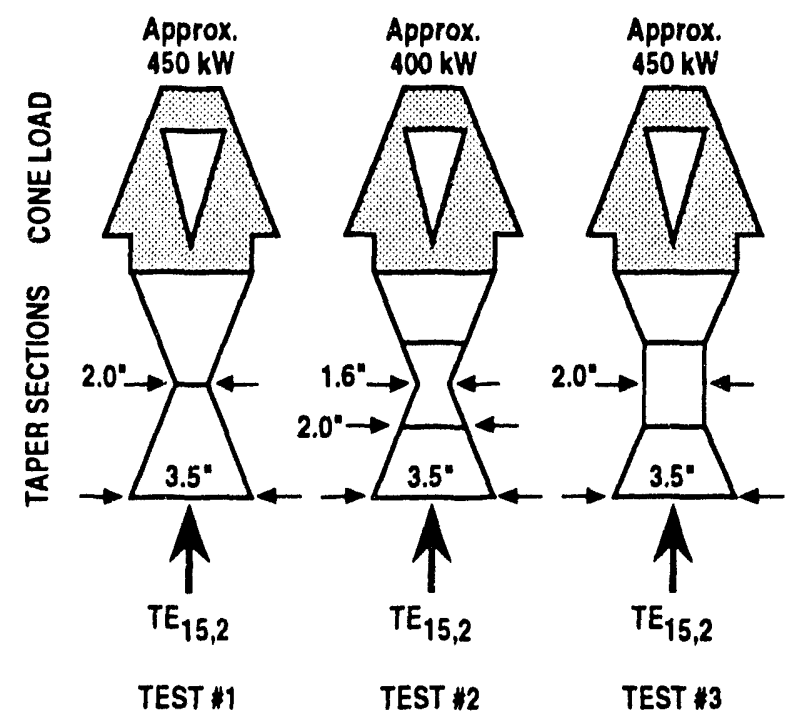

Fig. 3 Taper test configuration. alternate modes also increased (Fig. 4) and reached levels as high as $25 \%$ of the power in unwanted alternate modes. However, there were areas where approximately $90 \%$ of the gyrotron power is in the primary $\mathrm{TE}_{15.2}$ mode, unfortunately these seem to occur in a lower total power output range.

\section{Mode Converter Efficiency}

The GA mode converter was operated using standard calorimetric methods to determine the conversion efficiency of the primary $\mathrm{TE}_{15.2}$ mode and alternate modes compared with the total throughput power. Operational settings were adjusted to provide a high $\mathrm{TE}_{15.2}$ mode throughput power. Data was gathered with cathode current settings of $15 \mathrm{~A}, 17 \mathrm{~A}$, and approximately 19A (Fig. 5).

An University of Wisconsin advanced Vlasov type converter was made available to the DIII-D ECH program for testing. This quasi-optical converter had previously demonstrated a conversion efficiency of approximately $90 \%$ in low power laboratory testing, similar to the results of the GA mode converter laboratory testing $(\geq 90 \%)$. With the cathode current set at $18 \mathrm{~A}$, standard calorimetric methods were used to determine the output power in the primary $\mathrm{TE}_{15,2}$ mode and alternate modes. Figure 6 illustrates the total power versus the output power in the primary $\mathrm{TE}_{15,2}$ mode and alternate modes.

Both mode converters displayed the same trend consistent with a large fraction of non-primary $\mathrm{TE}_{15,2}$ mode being detected as the output power of the gyrotron is increased. Figure 7 illustrates the total power versus the mode power of both mode converters. From this data, it can be observed that the GA mode converter is more efficient when the throughput power is less than $400 \mathrm{~kW}$, which is where the highest mode purity is, and that the gyrotron seems less affected by the University of Wisconsin mode converter, probably its open geometry results in less reflected power back into the gyrotron.

\section{Collector Profile}

The key to achieving long pulse operation is the ability to spread the heat uniformly along the collector. Attempts were

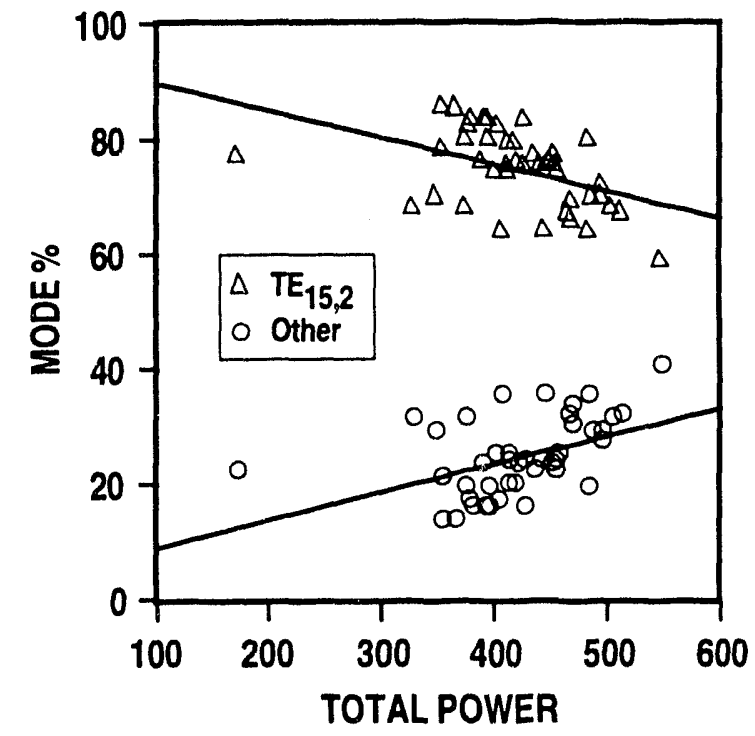

Fig. 4. Mode content versus total output power of the GA mode separation diagnestic. 


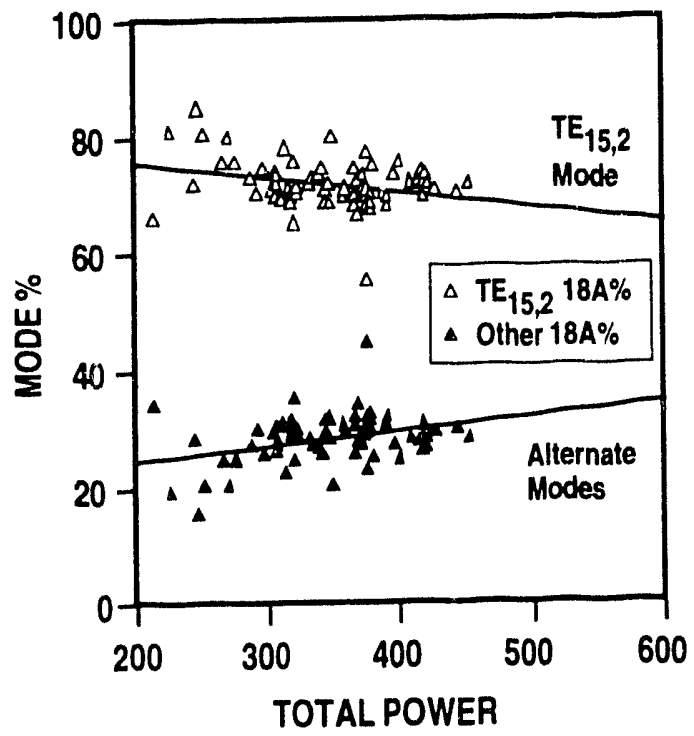

Fig. 5. Mode content versus total output power of the University of Wisconsin mode converter.

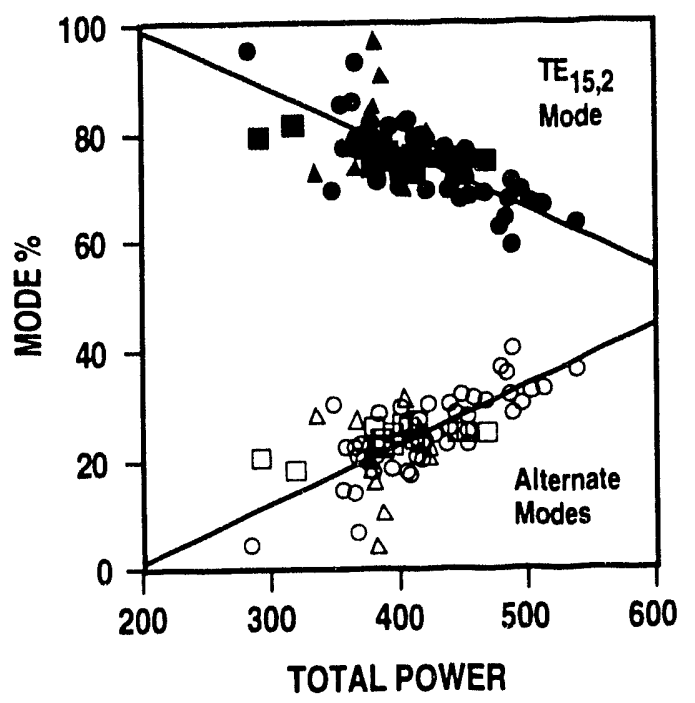

\begin{tabular}{|ll}
\hline$T E_{15,2} 15 A \%$ & $\square$ Other $15 A \%$ \\
$\Delta T E_{15,2} 17 A \%$ & $\Delta$ Other $17 A \%$ \\
$-T E_{15,2} 19 A \%$ & $\circ$ Other $19 A \%$
\end{tabular}

Fig. 6 Mode content versus total output power of the GA mode converter.

made to adjust the collector coil spreading magnets to spread the spent electron beam evenly along the collector. However, the operation of the gyrotron remained limited in pulse length due to hot spots in the collector region. Measurements showed unacceptable concentrations of power in elliptical patterns. The measured elliptical heat deposition pattern was determined to be resulting from radial magnetic fields caused by local iron distorting the fields from the super conducting magnet. Fields as low as 2-4 Gauss were the cause of the hot spots observed. A similar result was observed during testing at Varian. This observation lead to the realization that if a rotating radial magnetic field could be applied to the collector, the heat load could be spread more uniformly over the area of the collector. This

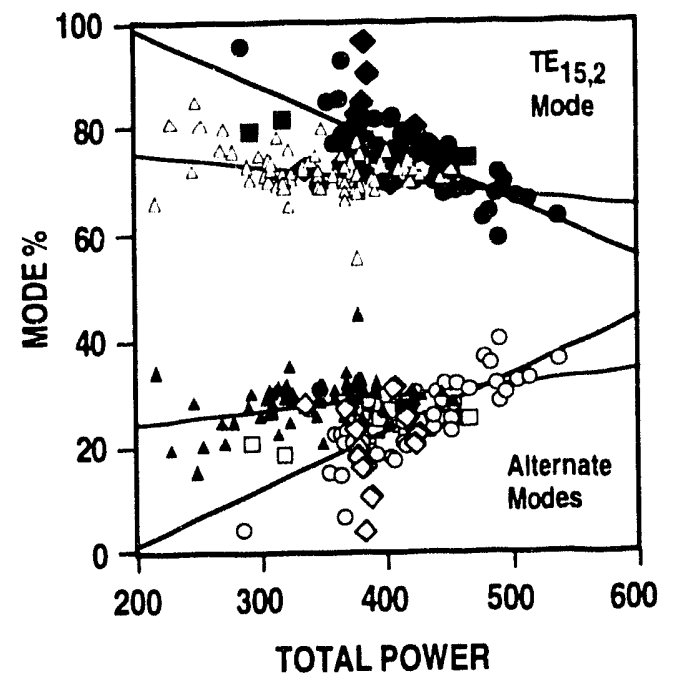

\begin{tabular}{|ccc|}
\hline \multicolumn{2}{|c}{ GENERAL ATOMICS } & U. OF WISCONSIN \\
$-\mathrm{TE}_{15,2} 15 \mathrm{~A} \%$ & $\square$ Other $15 \mathrm{~A} \%$ & $\triangle \mathrm{TE}_{15,2} 18 \mathrm{~A} \%$ \\
$-\mathrm{TE}_{15,2} 17 \mathrm{~A} \%$ & $\triangle$ Other $17 \mathrm{~A} \%$ & $\triangle$ Other $18 \mathrm{~A} \%$ \\
$-\mathrm{TE}_{15,2} 19 \mathrm{~A} \%$ & o Other $19 \mathrm{~A} \%$ & \\
\hline
\end{tabular}

Fig. 7. Mode content versus total outpower of both mode converters.

rotating radial field effectively spins the elliptical patterns around the collector. A gyrotron, with such a rotating coil, has shown a reduction by a factor of 3 in the peak collector heat load. This gyrotron has been operated at pulse lengths of $1.5 \mathrm{~s}$, and is now being commissioned at General Atomics.

\section{CONCLUSION}

The overall progress of the $110 \mathrm{GHz}$ ECH system during the first year of operation has been positive. Important results were obtained regarding transmission line component efficiency, and output mode purity. Through operations and testing, much information has been gained to increase system reliability and thereby provide an optimal system for future physics experiments on DIII-D. Continued testing is planned to further optimize the ECH system.

\section{ACKNOWLEDGMENT}

John Doane provided the transmission loss data for the 110 $\mathrm{GHz}$ waveguide system.

\section{REFERENCES}

[1] W.P. Cary, "110 GHz ECH on DIII-D: system overview and initial operation," in Proc. 14th IEEE/NPSS Symposium on Fusion Engineering, San Diego, California, September 30 through October 3, 1991, vol. 2, pp. 912-914.

[2] R.W. Callis, "110 GHz ECH heating system for DIII-D," in Proc. AIP Conf. on Radio Frequency Power in Plasmas, 1991, Charleston, South Carolina.

[3] C.P. Moeller, "2 MW $110 \mathrm{GHz}$ ECH heating system for DIIl-D," in Proc. 16th Symposium on Fusion Technology. vol. 2 , pp. 1040-1043. 

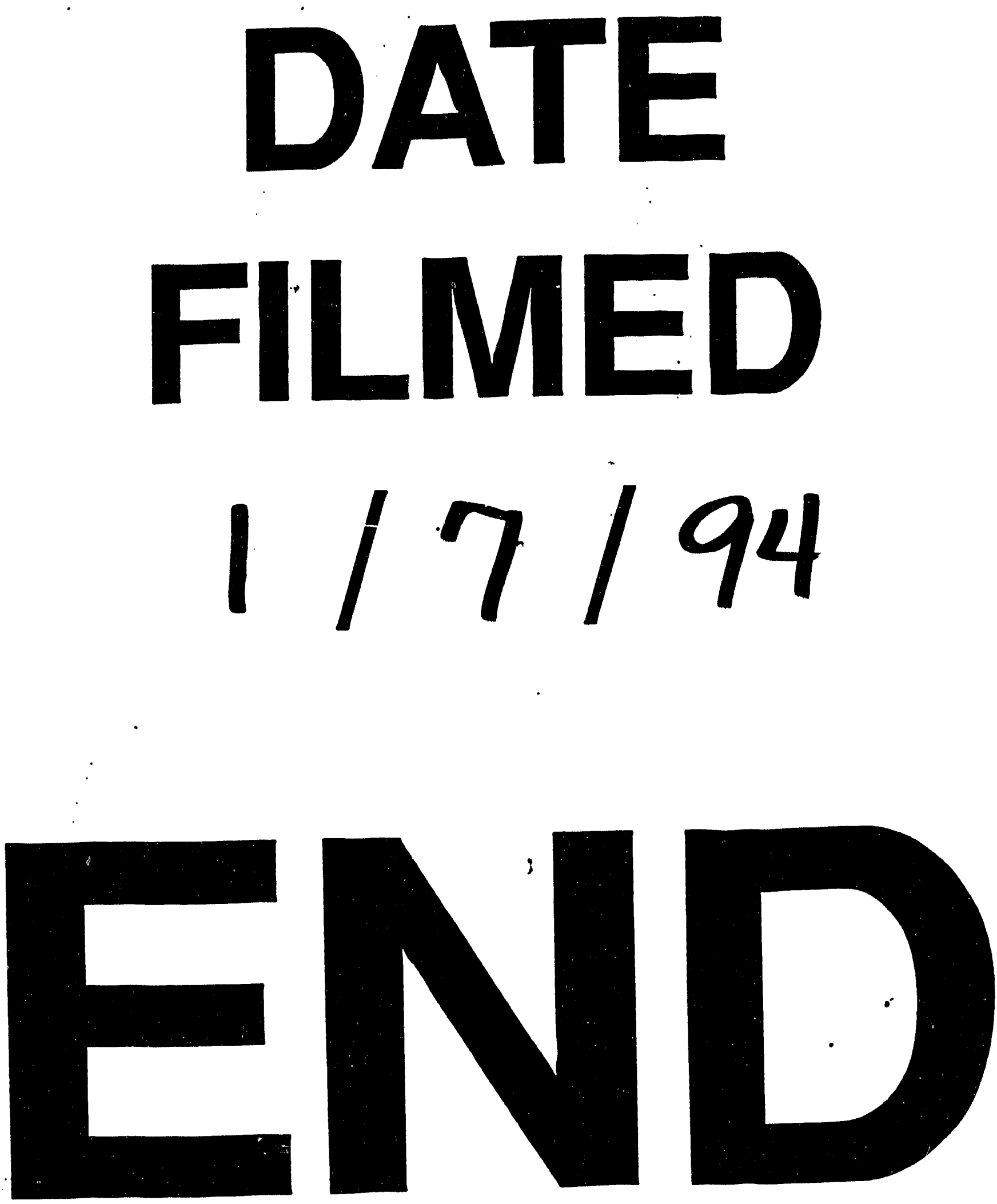
